Supporting Information

\title{
1,2,4-Triazolyl 5-Azaspiro[2.4]heptanes: Lead Identification and Early Lead Optimization of a New Series of Potent and Selective Dopamine D3 Receptor Antagonists.
}

Fabrizio Micheli ${ }^{*} a$, Alessia Bacchi ${ }^{\#}$, Simone Braggio ${ }^{a}$, Laura Castelletti ${ }^{a}$, Palmina Cavallini ${ }^{a}$, Paolo Cavanni ${ }^{a}$, Susanna Cremonesi ${ }^{a}$, Michele Dal Cin ${ }^{a}$, Aldo Feriani ${ }^{a}$, Sylvie Gheanne $^{a}$, Mahmoud Kajbaf ${ }^{a}$, Luciano Marchión, Selena Nola ${ }^{a}$, Beatrice Oliosi ${ }^{a}$, Annalisa Pellacani $^{a}$, Elisabetta Perdona' ${ }^{a}$, Anna Sava ${ }^{a}$, Teresa Semeraro ${ }^{a}$ Luca Tarsi ${ }^{a}$, Silvia Tomelleri ${ }^{a}$, Andrea Wong ${ }^{a}$, Filippo Visentini ${ }^{a}$, Laura Zonzini ${ }^{a}$, and Christian Heidbreder ${ }^{b}$.

a Aptuit s.r.l., Via Fleming 4, 37135 Verona (Italy), ' Indivior Inc, The Fairfax Building, 10710 Midlothian Turnpike, Suite 430, Richmond VA 23235, USA, \# Dipartimento di Chimica, Universita' di Parma, Viale delle Scienze, 17A - Campus, I-43124 Parma, Italy

Content:

S2 - Experimental for key intermediates including NMR interpretation

S13 - checkCIF/PLATON report 


\section{Cis and Trans 5-benzyl-1-[2-(trifluoromethyl)phenyl]-5-azaspiro[2.4]heptane-4,6-dione}

To a solution of \{[2-(trifluoromethyl)phenyl]methylidene $\}$ hydrazine $(0.935 \mathrm{~g}, 4.97 \mathrm{mmol})$ in dioxane $(6 \mathrm{~mL})$ at $10{ }^{\circ} \mathrm{C}, \mathrm{MnO}_{2}(4.32 \mathrm{~g}, 49.7 \mathrm{mmol})$ was added portionwise. The resulting mixture was stirred at RT for $30 \mathrm{~min}$, then it was filtered over a pad of Celite washing with dioxane $(15 \mathrm{~mL})$ directly into a solution of 1-benzyl-3-methylidenepyrrolidine-2,5-dione $(1 \mathrm{~g}$, $4.97 \mathrm{mmol}$ ) in dioxane $(3 \mathrm{~mL})$. The resulting orange/red solution was left stirring at $\mathrm{RT} \mathrm{O} / \mathrm{N}$. Solvent was removed and the residue was purified by FC on silica gel (eluent: cHex to $30 \%$ EtOAc, then to EtOAc 100\%) affording a mixture of both diastereomers that were separated via chiral Prep HPLC

\section{Preparative chromatography:}

Column

Mobile phase

Flow rate $(\mathrm{ml} / \mathrm{min})$

DAD detection

Loop

Injection
Chiralpak AD-H $(25 \times 2.0 \mathrm{~cm}), 5 \mu$

n-Hexane/(Ethanol/Methanol $+0.1 \%$ isopropylamine) $80 / 20 \% \mathrm{v} / \mathrm{v}$

$18 \mathrm{ml} / \mathrm{min}$

$220 \mathrm{~nm}$

$330 \mu \mathrm{L}$

$41 \mathrm{mg} /$ injection

\section{Affording:}

(1S,3S/1R,3R)-5-benzyl-1-[2-(trifluoromethyl)phenyl]-5-azaspiro[2.4]heptane-4,6-dione

(Diastereoisomer 2, TRANS): $425 \mathrm{mg}, 100 \%$ purity, r.t. $1.20 \mathrm{~min}$

(1R,3S/1S,3R)-5-benzyl-1-[2-(trifluoromethyl)phenyl]-5-azaspiro[2.4]heptane-4,6-dione

(Diastereoisomer 1, CIS): $531 \mathrm{mg}, 100 \%$ purity, r.t. $1.17 \mathrm{~min}$ 


\section{Trans 5-benzyl-1-[2-(trifluoromethyl)phenyl]-5-azaspiro[2.4]heptane-4,6-dione}

${ }^{1} \mathrm{H}$ NMR Assignment

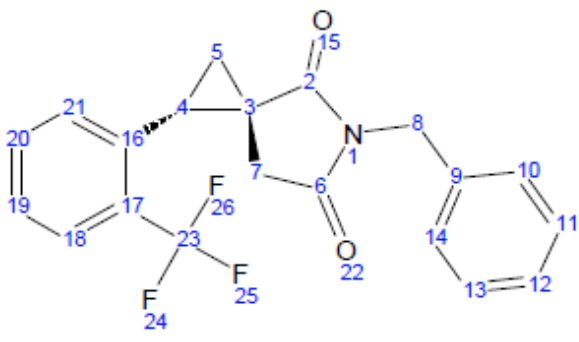

\begin{tabular}{|c|c|c|c|c|}
\hline Shift1 (ppm) & X's & Multiplicity & $\mathrm{J}(\mathrm{Hz})$ & Atom1 \\
\hline 1.78 & 1 & $\mathrm{~d}$ & 18.59 & $7<">$ \\
\hline 1.84 & 1 & $\mathrm{dd}$ & $9.29,5.38$ & $5<">$ \\
\hline 2.03 & 1 & $\mathrm{t}$ & 6.36 & $5<>$ \\
\hline 2.76 & 1 & $\mathrm{~d}$ & 18.59 & $7<>$ \\
\hline 2.91 & 1 & $\mathrm{t}$ & 7.83 & 4 \\
\hline 4.62 & 2 & $\mathrm{~m}$ & - & 8 \\
\hline 7.24 & 2 & $\mathrm{~m}$ & - & 10,14 \\
\hline 7.28 & 1 & $\mathrm{~m}$ & - & 12 \\
\hline 7.33 & 2 & $\mathrm{~m}$ & - & 11,13 \\
\hline 7.42 & 1 & $\mathrm{~d}$ & 7.80 & 21 \\
\hline 7.53 & 1 & $\mathrm{t}$ & 7.80 & 19 \\
\hline 7.68 & 1 & $\mathrm{t}$ & 7.80 & 20 \\
\hline 7.76 & 1 & $\mathrm{~d}$ & 7.80 & 18 \\
\hline
\end{tabular}

NMR Spectrometer Varian D.Drive 500 (L4019)

NMR Spectra A_1712_85_4_500_PROTON_01

A_1712_85_4_500_gCOSY_01

A_1712_85_4_500_gHSQCAD_01

A_1712_85_4_500_ROESY_01

${ }^{1} \mathrm{H}$ NMR $\left(500 \mathrm{MHz}\right.$, DMSO-d $\left.d_{6}\right) \delta \mathrm{ppm} 1.78(\mathrm{~d}, J=18.59 \mathrm{~Hz}, 1 \mathrm{H}), 1.84(\mathrm{dd}, J=9.29,5.38 \mathrm{~Hz}, 1 \mathrm{H}), 2.03(\mathrm{t}, J=6.36 \mathrm{~Hz}, 1 \mathrm{H}), 2.76(\mathrm{~d}, J=18.59 \mathrm{~Hz}, 1 \mathrm{H}), 2.91$ $(\mathrm{t}, J=7.83 \mathrm{~Hz}, 1 \mathrm{H}), 4.55-4.68(\mathrm{~m}, 2 \mathrm{H}), 7.22-7.26(\mathrm{~m}, 2 \mathrm{H}), 7.26-7.30(\mathrm{~m}, 1 \mathrm{H}), 7.30-7.36(\mathrm{~m}, 2 \mathrm{H}), 7.42(\mathrm{~d}, J=7.80 \mathrm{~Hz}, 1 \mathrm{H}), 7.53(\mathrm{t}, J=7.80 \mathrm{~Hz}, 1 \mathrm{H})$ $7.68(\mathrm{t}, J=7.80 \mathrm{~Hz}, 1 \mathrm{H}), 7.76(\mathrm{~d}, J=7.80 \mathrm{~Hz}, 1 \mathrm{H})$<smiles></smiles>
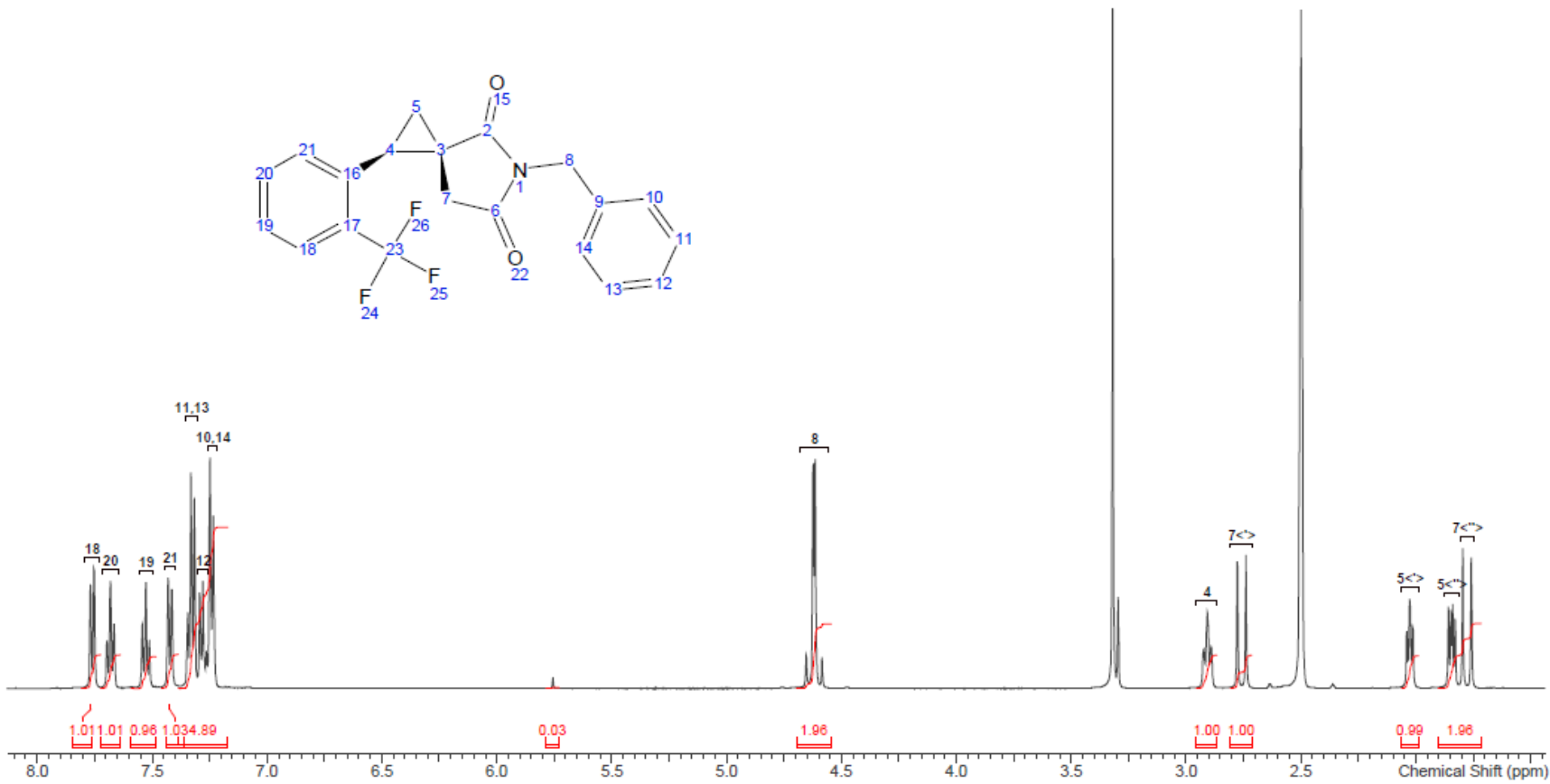


\section{Cis 5-benzyl-1-[2-(trifluoromethyl)phenyl]-5-azaspiro[2.4]heptane-4,6-dione}

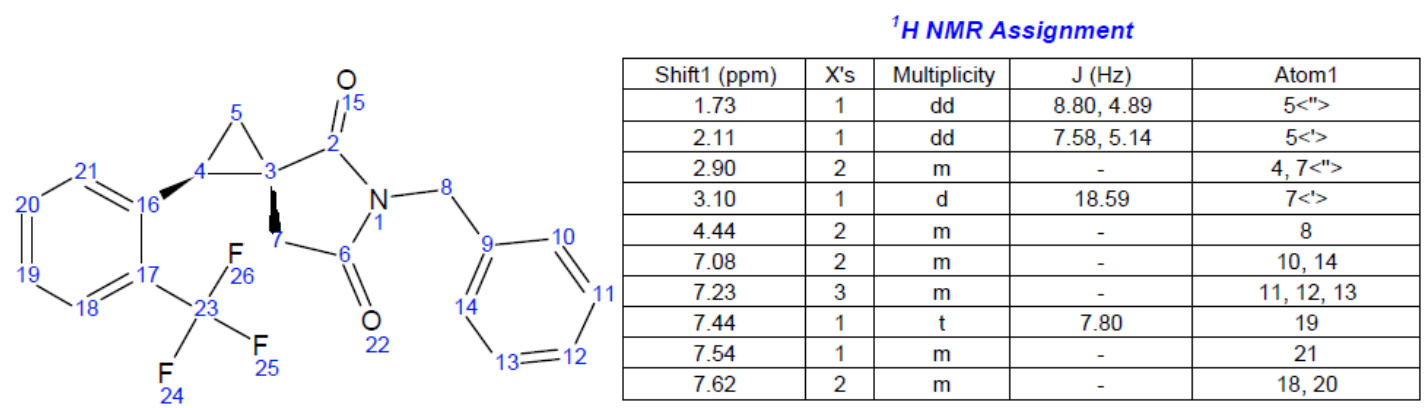

NMR Spectrometer Varian D.Drive 500 (L4019)

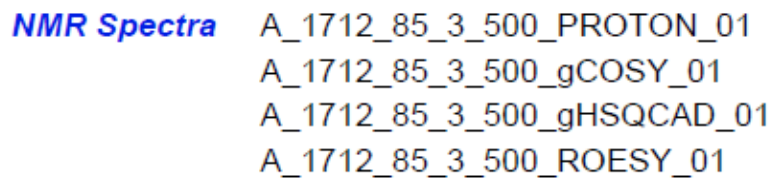

NMR Spectra A_1712_85_3_500_PROTON_01

A_1712_85_3_500_gCOSY_01

A_1712_85_3_500_gHSQCAD_01

A_1712_85_3_500_ROESY_01

${ }^{1} \mathrm{H}$ NMR $\left(500 \mathrm{MHz}, D M S O-d_{6}\right) \delta \mathrm{ppm} 1.73(\mathrm{dd}, J=8.80,4.89 \mathrm{~Hz}, 1 \mathrm{H}), 2.11(\mathrm{dd}, J=7.58,5.14 \mathrm{~Hz}, 1 \mathrm{H}), 2.82-2.96(\mathrm{~m}, 2 \mathrm{H}), 3.10(\mathrm{~d}, J=18.59 \mathrm{~Hz}, 1 \mathrm{H}), 4.33$ - $4.56(\mathrm{~m}, 2 \mathrm{H}), 7.04-7.11(\mathrm{~m}, 2 \mathrm{H}), 7.19-7.28(\mathrm{~m}, 3 \mathrm{H}), 7.44(\mathrm{t}, J=7.80 \mathrm{~Hz}, 1 \mathrm{H}), 7.51-7.56(\mathrm{~m}, 1 \mathrm{H}), 7.57-7.67(\mathrm{~m}, 2 \mathrm{H})$

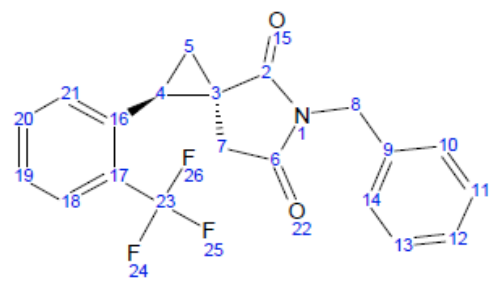

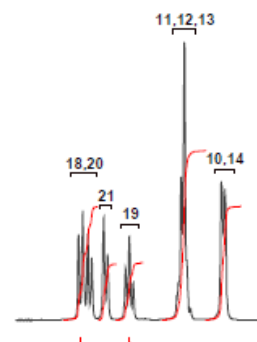

2.020 .801 .023 .012 .02

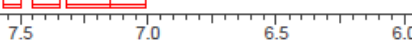

.07
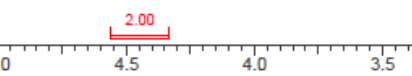

1.02 .1 .98 


\section{Trans 5-benzyl-1-[4-fluoro-2-(trifluoromethyl)phenyl]-5-azaspiro[2.4]heptane-4,6-dione}

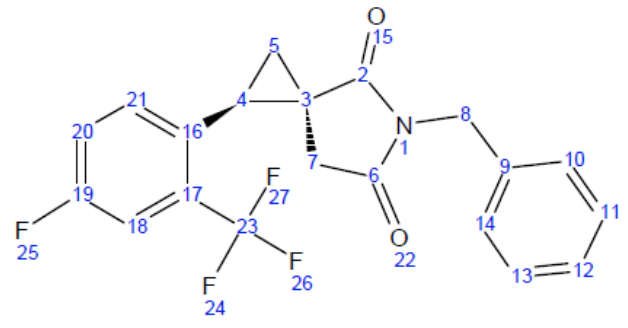

${ }^{1}$ H NMR Assignment

\begin{tabular}{|c|c|c|c|c|}
\hline Shift1 $(\mathrm{ppm})$ & X's & Multiplicity & $\mathrm{J}(\mathrm{Hz})$ & Atom1 \\
\hline 1.90 & 1 & $\mathrm{dd}$ & $9.29,5.38$ & $5\left\langle^{\prime \prime}\right\rangle$ \\
\hline 1.97 & 1 & $\mathrm{~m}$ & - & $5\left\langle^{\prime}\right\rangle$ \\
\hline 2.01 & 1 & $\mathrm{~m}$ & - & $7\left\langle^{\prime \prime}\right\rangle$ \\
\hline 2.66 & 1 & $\mathrm{~d}$ & 18.59 & $7\left\langle{ }^{\prime}\right\rangle$ \\
\hline 3.00 & 1 & $\mathrm{t}$ & 8.07 & 4 \\
\hline 4.68 & 2 & $\mathrm{~m}$ & - & 8 \\
\hline 7.29 & 5 & $\mathrm{~m}$ & - & $14,13,12,11,10$ \\
\hline 7.47 & 2 & $\mathrm{~m}$ & - & 21,20 \\
\hline 7.56 & 1 & $\mathrm{dd}$ & $9.05,2.69$ & 18 \\
\hline
\end{tabular}

NMR Spectrometer Varian D.Drive 500 (L4019);

Varian D.Drive 400 (L4019)

\section{NMR Spectra Acetone-d6:}

A_1745_57_4_500_PROTON_01

A_1745_57_4_500_gCOSY_01

A_1745_57_4_500_gHSQCAD_01

A_1745_57_4_500_ROESY_01

Methanol-d4

A 1745_57_4400_PROTON 01

A_1745_57_4_400_NOESY1D_01

A_1745_57_4_400_NOESY1D_02

${ }^{1} \mathrm{H}$ NMR $(500 \mathrm{MHz}$, Acetone) $\delta \mathrm{ppm} 1.90(\mathrm{dd}, J=9.29,5.38 \mathrm{~Hz}, 1 \mathrm{H}), 1.94-1.98(\mathrm{~m}, 1 \mathrm{H}), 1.99-2.04(\mathrm{~m}, 1 \mathrm{H}), 2.66(\mathrm{~d}, J=18.59 \mathrm{~Hz}, 1 \mathrm{H}), 3.00(\mathrm{t}, J=8.07$ $\mathrm{Hz}, 1 \mathrm{H}), 4.63-4.73(\mathrm{~m}, 2 \mathrm{H}), 7.24-7.37(\mathrm{~m}, 5 \mathrm{H}), 7.42-7.53(\mathrm{~m}, 2 \mathrm{H}), 7.56(\mathrm{dd}, J=9.05,2.69 \mathrm{~Hz}, 1 \mathrm{H})$

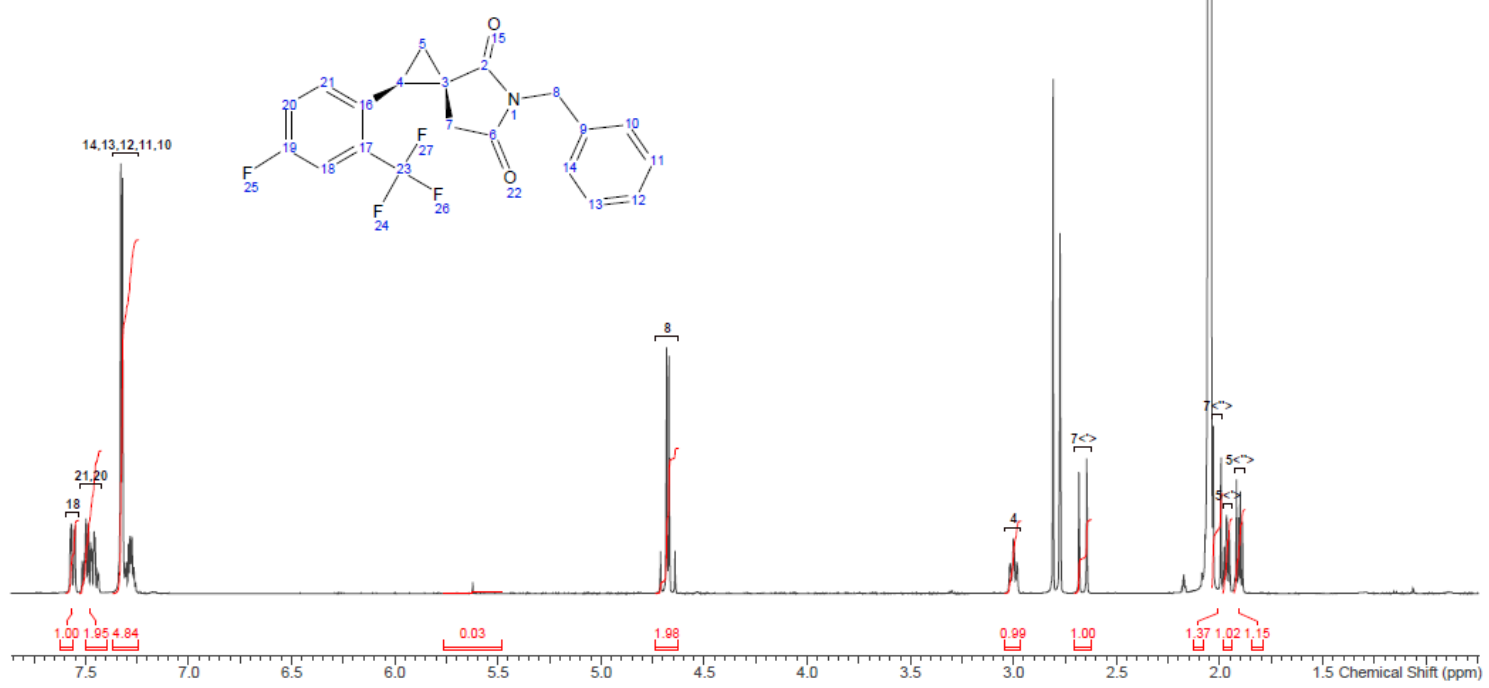


Cis 5-benzyl-1-[4-fluoro-2-(trifluoromethyl)phenyl]-5-azaspiro[2.4]heptane-4,6-dione<smiles>O=C1N(Cc2ccncc2)S(=O)C[C@]12C[C@H]2c1ccc(F)c[p+]1C(F)(F)F</smiles>

'H NMR Assignment
\begin{tabular}{|c|c|c|c|c|}
\hline Shift1 (ppm) & $X^{\prime}$ s & Multiplicity & $\mathrm{J}(\mathrm{Hz})$ & Atom 1 \\
\hline 1.79 & 1 & $\mathrm{dd}$ & $8.80,5.00$ & $5<">$ \\
\hline 2.14 & 1 & $\mathrm{dd}$ & $7.34,5.00$ & $5<'>$ \\
\hline 2.93 & 2 & $\mathrm{~m}$ & - & $4,7<">$ \\
\hline 3.04 & 1 & $\mathrm{~m}$ & - & $7<'>$ \\
\hline 4.47 & 1 & $\mathrm{~m}$ & - & $\left.8<{ }^{\prime}\right\rangle$ \\
\hline 4.55 & 1 & $\mathrm{~m}$ & - & $8<'>$ \\
\hline 7.21 & 5 & $\mathrm{~m}$ & - & $10,11,12,13,14$ \\
\hline 7.40 & 2 & $\mathrm{~m}$ & - & 18,20 \\
\hline 7.61 & 1 & $\mathrm{dd}$ & $8.56,5.62$ & 21 \\
\hline
\end{tabular}

NMR Spectrometer Varian D.Drive 500 (L4019)

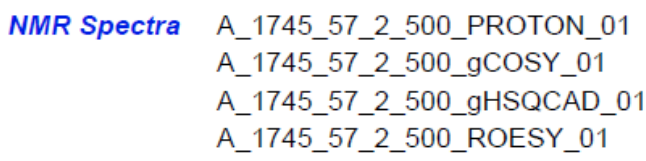

${ }^{1} \mathrm{H}$ NMR $(500 \mathrm{MHz}$, Acetone) $\delta \mathrm{ppm} 1.79(\mathrm{dd}, J=8.80,5.00 \mathrm{~Hz}, 1 \mathrm{H}), 2.14(\mathrm{dd}, J=7.34,5.00 \mathrm{~Hz}, 1 \mathrm{H}), 2.88-2.97(\mathrm{~m}, 2 \mathrm{H}), 3.00-3.08(\mathrm{~m}, 1 \mathrm{H}), 4.43-4.51$ $(\mathrm{m}, 1 \mathrm{H}), 4.51-4.58(\mathrm{~m}, 1 \mathrm{H}), 7.15-7.28(\mathrm{~m}, 5 \mathrm{H}), 7.34-7.46(\mathrm{~m}, 2 \mathrm{H}), 7.61(\mathrm{dd}, J=8.56,5.62 \mathrm{~Hz}, 1 \mathrm{H})$
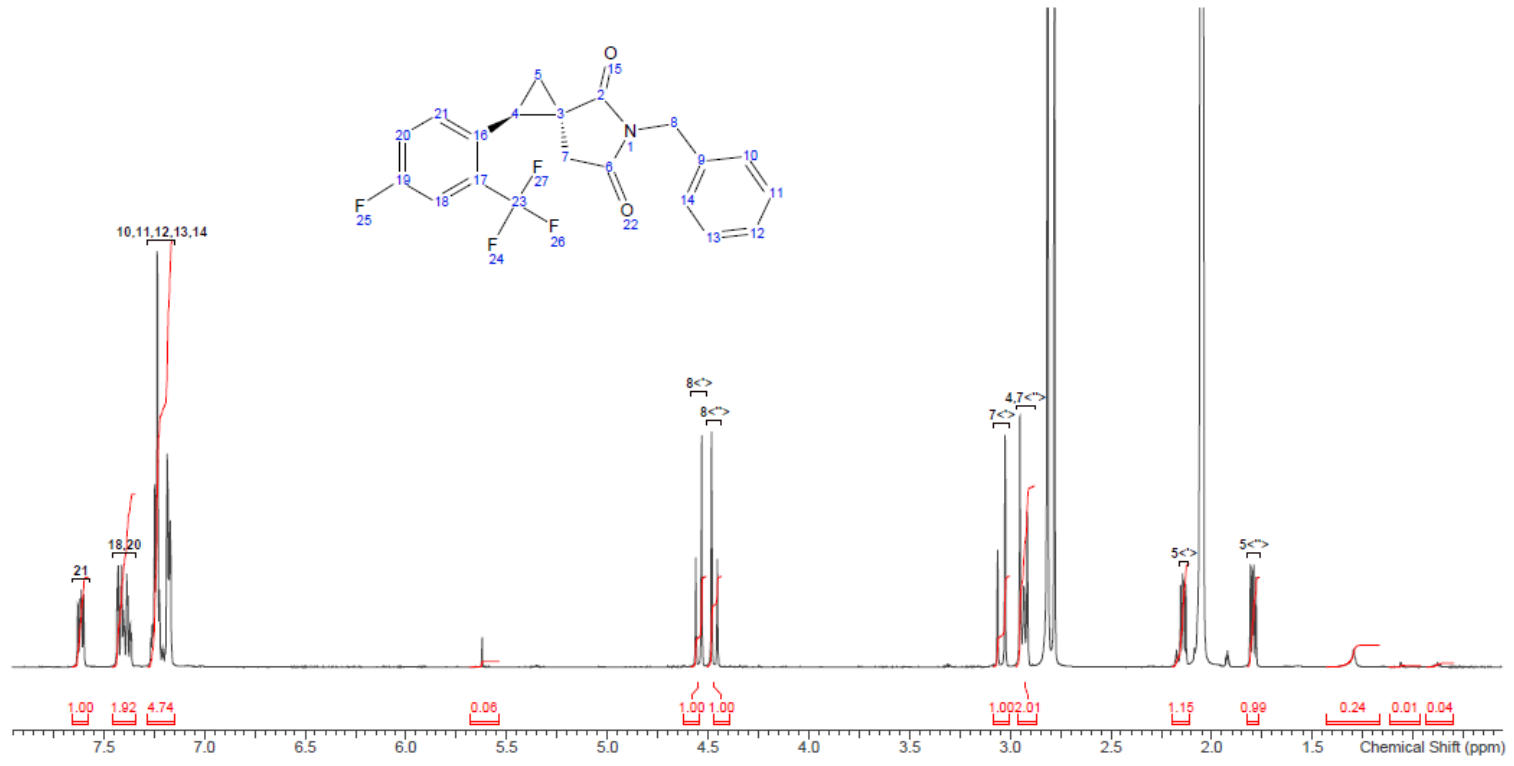


\section{Trans 1-[4-(trifluoromethyl)phenyl]-5-azaspiro[2.4]heptane}

\section{Step a:}

(1S,3S/1R,3R)-5-benzyl-1-[4-(trifluoromethyl)phenyl]-5-azaspiro[2.4]heptane-4,6-dione

(Diastereoisomer 1, TRANS, $0.91 \mathrm{~g}, 2.53 \mathrm{mmol}$ ) was dissolved in THF (15 mL) and $\mathrm{LiAlH}_{4}$ $1 \mathrm{M}$ in THF (5.06 mL, $5.06 \mathrm{mmol}$ ) was added dropwise. The resulting orange solution was heated at reflux for $1 \mathrm{~h}$. Then it was cooled with an ice bath and quenched with $\mathrm{Na}_{2} \mathrm{SO}_{4} * 10$ $\mathrm{H}_{2} \mathrm{O}$ until gas evolution ceased. The mixture was filtered over a pad of Celite washing with EtOAc, and the solution was concentrated to afford (1S,3S/1R,3R)-5-benzyl-1-[4(trifluoromethyl)phenyl]-5-azaspiro[2.4]heptane (TRANS, $810 \mathrm{mg}$ ) as oil that was used as such in the next step.

\section{Step b:}

(1S,3S/1R,3R)-5-benzyl-1-[4-(trifluoromethyl)phenyl]-5-azaspiro[2.4]heptane (TRANS, 810 $\mathrm{mg}$ from step a) was dissolved in $\mathrm{MeOH}(20 \mathrm{~mL})$ under $\mathrm{N}_{2}$ and ammonium formate $(1.55 \mathrm{~g}$, $24.4 \mathrm{mmol})$ was added. After 2 cycles vacuum $/ \mathrm{N}_{2} \mathrm{Pd} / \mathrm{C}(0.25 \mathrm{~g})$ was added. The resulting mixture was stirred at reflux for $1 \mathrm{~h}$. After cooling down to RT, it was filtered over a pad of Celite, the solvent was evaporated and the residue was charged on SCX cartridge (eluting with $1 \mathrm{~N} \mathrm{NH}_{3}$ in $\mathrm{MeOH}$ ) to afford, after evaporation, (1S,3S/1R,3R)-1-[4(trifluoromethyl)phenyl]-5-azaspiro[2.4]heptane (TRANS, $495 \mathrm{mg}, \mathrm{y}=81 \%$ ) as pale yellow oil. 
Trans 1-[4-(trifluoromethyl)phenyl]-5-azaspiro[2.4]heptane

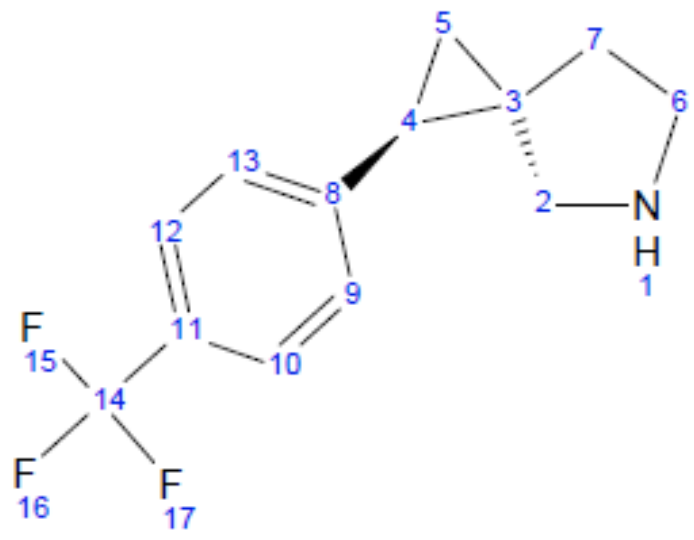

${ }^{1} \mathrm{H}$ NMR Assignment

\begin{tabular}{|c|c|c|c|c|}
\hline Shift1 (ppm) & $X^{\prime}$ s & Type & $J(H z)$ & Atom1 \\
\hline 1.26 & 2 & $\mathrm{~m}$ & - & 5 \\
\hline 1.44 & 1 & $\mathrm{~s}$ & - & $7\langle>$ \\
\hline 1.85 & 1 & $\mathrm{~m}$ & - & $7<>$ \\
\hline 2.22 & 1 & $\mathrm{dd}$ & $8.8,6.4$ & 4 \\
\hline 3.03 & 1 & $\mathrm{~m}$ & - & $6<\rangle$ \\
\hline 3.08 & 2 & $\mathrm{~s}$ & - & 2 \\
\hline 3.15 & 1 & $\mathrm{~m}$ & - & $8<\rangle$ \\
\hline 7.24 & 2 & $\mathrm{~d}$ & 8.3 & 13,9 \\
\hline 7.54 & 2 & $\mathrm{~d}$ & 8.3 & 12,10 \\
\hline
\end{tabular}

NMR Spectra A_1598_75_1_500_PROTON_01

A_1598_75_1_500_gCOSY_01

A 1598 75_1_500_gHSQCAD_01

A_1598_75_1_500_gHMBCAD_01

A_1598_75_1_500_ROESY_01

${ }^{1} \mathrm{H}$ NMR $(500 \mathrm{MHz}$, CHLOROFORM- $d$ ) $\delta$ ppm $7.54(\mathrm{~d}, J=8.3 \mathrm{~Hz}, 2 \mathrm{H}), 7.24(\mathrm{~d}, J=8.3 \mathrm{~Hz}, 2 \mathrm{H}), 3.11-3.18(\mathrm{~m}, 1 \mathrm{H}), 3.08(\mathrm{~s}, 2 \mathrm{H}), 2.99-3.06(\mathrm{~m}, 1 \mathrm{H})$, $2.22(\mathrm{dd}, J=8.8,6.4 \mathrm{~Hz}, 1 \mathrm{H}), 1.61-1.70(\mathrm{~m}, 1 \mathrm{H}), 1.44(\mathrm{~s}, 1 \mathrm{H}), 1.20-1.31(\mathrm{~m}, 2 \mathrm{H})$

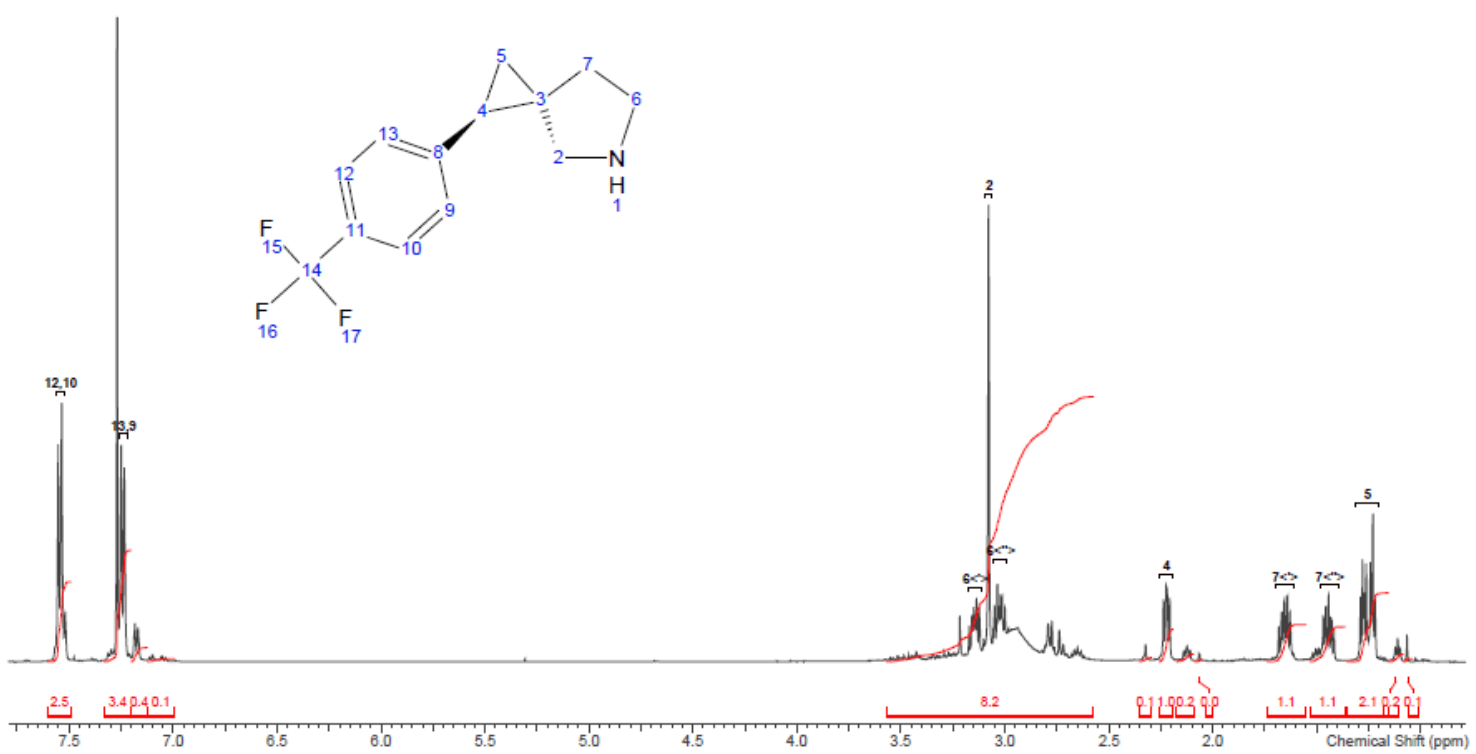




\section{Cis 1-[4-(trifluoromethyl)phenyl]-5-azaspiro[2.4]heptane}

\section{Step a:}

(1S,3R/1R,3S)-5-benzyl-1-[4-(trifluoromethyl)phenyl]-5-azaspiro[2.4]heptane-4,6-dione

(Diastereoisomer 2, CIS, $7.5 \mathrm{~g}, 20.87 \mathrm{mmol})$ was dissolved in THF $(120 \mathrm{~mL})$ and $\mathrm{LiAlH}_{4} 1 \mathrm{M}$ in THF $(15.65 \mathrm{~mL}, 15.65 \mathrm{mmol})$ was added dropwise at $0{ }^{\circ} \mathrm{C}$. The resulting orange solution was heated at reflux for $1 \mathrm{~h}$. Then it was cooled with an ice bath and quenched with $\mathrm{Na}_{2} \mathrm{SO}_{4}$ * $10 \mathrm{H}_{2} \mathrm{O}$ until gas evolution ceased. The mixture was filtered over a pad of Celite washing with EtOAc, and the solution was concentrated to afford (1R,3S/1S,3R)-5-benzyl-1-[4(trifluoromethyl)phenyl]-5-azaspiro[2.4]heptane (CIS, $6.9 \mathrm{~g}$ ) as oil that was used as such in the next step.

\section{Step b:}

(1R,3S/1S,3R)-5-benzyl-1-[4-(trifluoromethyl)phenyl]-5-azaspiro[2.4]heptane (CIS, $6.9 \mathrm{~g}$, from step a) was dissolved in $\mathrm{MeOH}(200 \mathrm{~mL})$ under $\mathrm{N}_{2}$ and ammonium formate $(6.58 \mathrm{~g}$, $104.35 \mathrm{mmol})$ was added. Then $\mathrm{Pd} / \mathrm{C}(800 \mathrm{mg})$ was added. The resulting mixture was stirred at reflux for $5 \mathrm{hrs}$. After cooling it was filtered over a pad of Celite, the solvent was evaporated and $12 \mathrm{~mL}$ of $\mathrm{HCl} \sim 1.25 \mathrm{M}$ in $\mathrm{MeOH}$ were added. Solvent was eliminated under reduced pressure and the residue was loaded on a SCX cartridge washing with $\mathrm{MeOH}$ and eluting with $\mathrm{NH}_{3} 1 \mathrm{M}$ in $\mathrm{MeOH}$. Solvent was eliminated under reduced pressure affording (1R,3S/1S,3R)-1-[4-(trifluoromethyl)phenyl]-5-azaspiro[2.4]heptane (CIS, $3.89 \mathrm{~g}, \mathrm{y}=77 \%$ ). 


\section{Cis 1-[4-(trifluoromethyl)phenyl]-5-azaspiro[2.4]heptane}

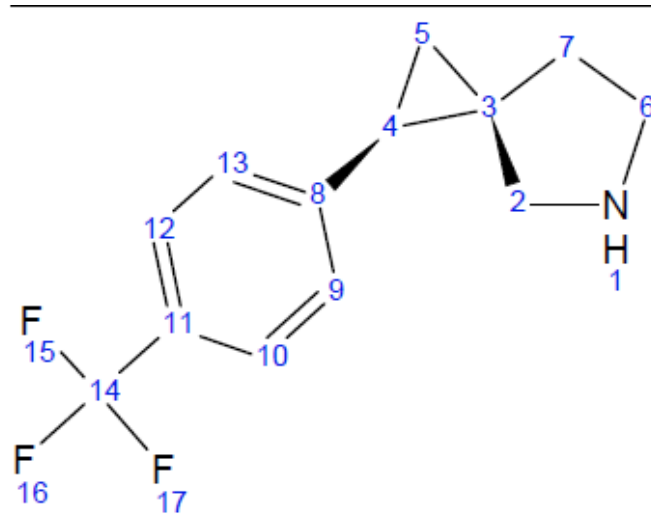

1 H NMR Assignment
\begin{tabular}{|c|c|c|c|c|}
\hline Shift1 $(\mathrm{ppm})$ & X's & Type & $\mathrm{J}(\mathrm{Hz})$ & Atom1 \\
\hline 1.19 & 1 & $\mathrm{~m}$ & - & $5<">$ \\
\hline 1.28 & 1 & $\mathrm{~m}$ & - & $5<>$ \\
\hline 1.95 & 2 & $\mathrm{~m}$ & - & 7 \\
\hline 2.23 & 1 & $\mathrm{dd}$ & $8.3,6.8$ & 4 \\
\hline 2.56 & 1 & $\mathrm{~d}$ & 11.2 & $2<">$ \\
\hline 2.74 & 1 & $\mathrm{~d}$ & 11.2 & $\left.2<{ }^{\prime}\right\rangle$ \\
\hline 3.13 & 2 & $\mathrm{~m}$ & - & 6 \\
\hline 7.19 & 2 & $\mathrm{~d}$ & 8.3 & 13,9 \\
\hline 7.53 & 2 & $\mathrm{~d}$ & 8.3 & 12,10 \\
\hline
\end{tabular}

NMR Spectrometer Varian D.Drive 500 (L4019)

NMR Spectra A 1598771500 PROTON 01

A_1598_77_1_500_gCOSY_01

A_1598_77_1_500_gHSQCAD_01

A_1598_77_1_500_gHMBCAD_01

A_1598_77_1_500_ROESY_01

${ }^{1} \mathrm{H}$ NMR $(500 \mathrm{MHz}$, CHLOROFORM-d) $\delta \mathrm{ppm} 7.53$ (d, $J=8.3 \mathrm{~Hz}, 2 \mathrm{H}), 7.19$ (d, $\left.J=8.3 \mathrm{~Hz}, 2 \mathrm{H}\right), 3.08-3.19$ (m, $\left.2 \mathrm{H}\right), 2.74$ (d, $\left.J=11.2 \mathrm{~Hz}, 1 \mathrm{H}\right), 2.56$ (d, $J=11.2 \mathrm{~Hz}, 1 \mathrm{H}), 2.23$ (dd, $J=8.3,6.8 \mathrm{~Hz}, 1 \mathrm{H}), 1.89-2.02(\mathrm{~m}, 2 \mathrm{H}), 1.26-1.31(\mathrm{~m}, 1 \mathrm{H}), 1.17-1.21(\mathrm{~m}, 1 \mathrm{H})$

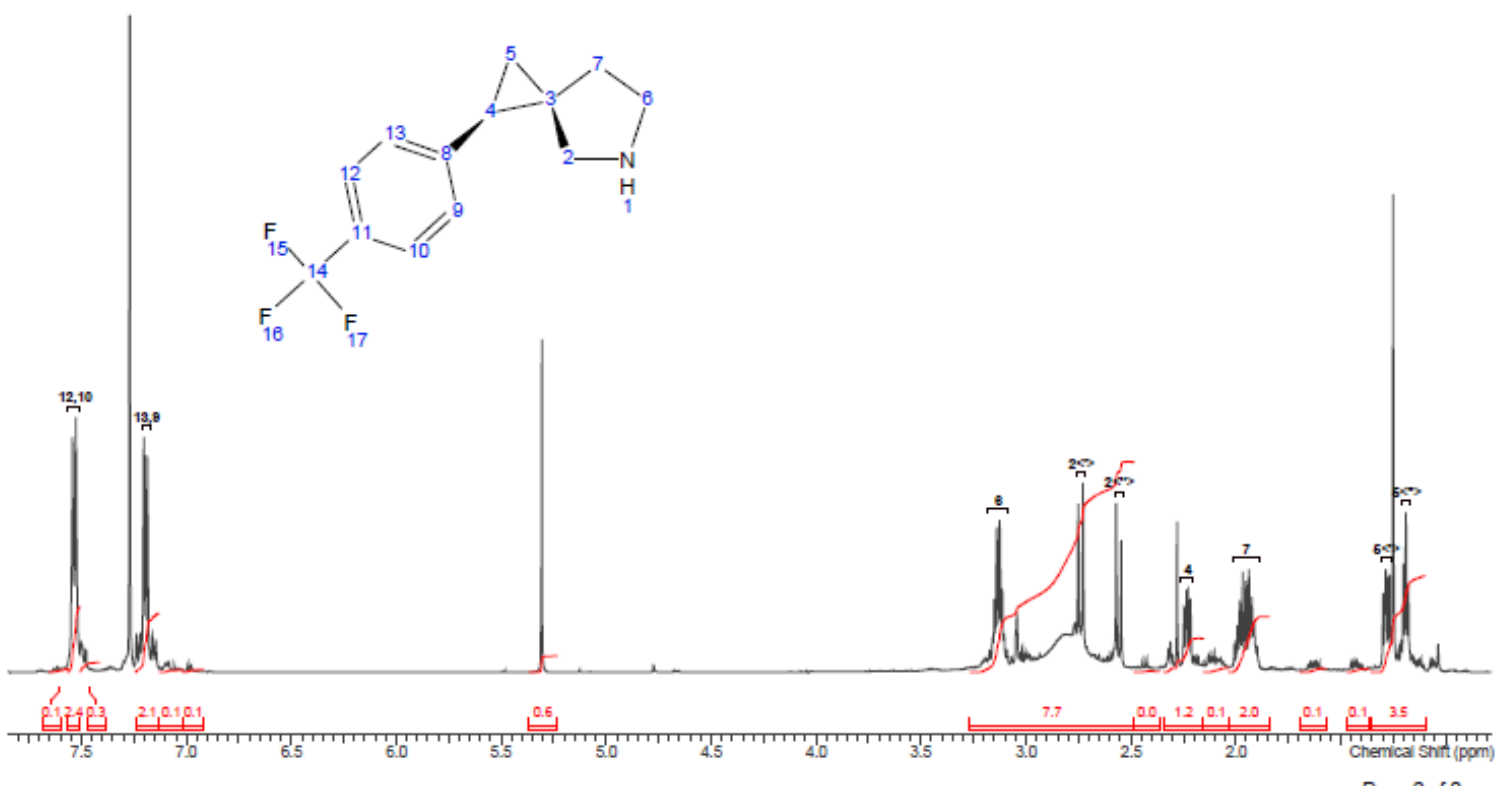




\section{Trans 1-[2-fluoro-4-(trifluoromethyl)phenyl]-5-azaspiro[2.4]heptane}

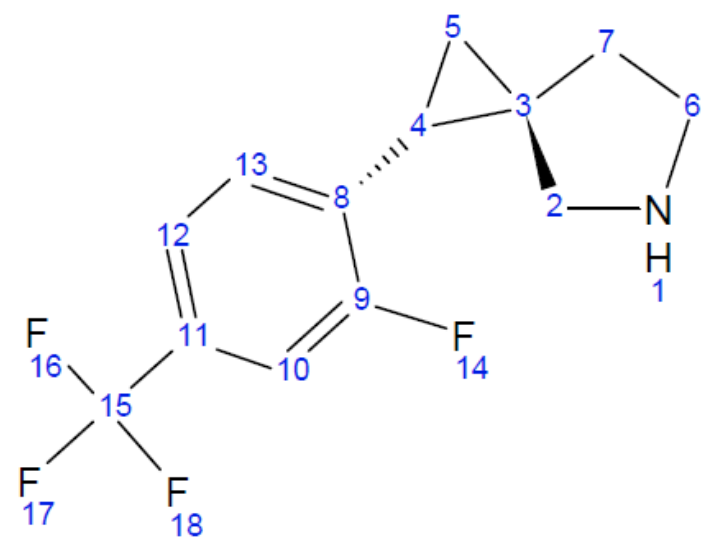

${ }^{1}$ H NMR Assignment

\begin{tabular}{|c|c|c|c|c|}
\hline Shift1 $(\mathrm{ppm})$ & $\mathrm{X}$ 's & Type & $\mathrm{J}(\mathrm{Hz})$ & Atom1 \\
\hline 1.20 & 1 & $\mathrm{~m}$ & - & $5\langle "\rangle$ \\
\hline 1.28 & 2 & $\mathrm{~m}$ & - & $7\langle "\rangle, 5\left\langle{ }^{\prime}\right\rangle$ \\
\hline 1.52 & 1 & $\mathrm{~m}$ & - & $7\left\langle{ }^{\prime}\right\rangle$ \\
\hline 2.21 & 1 & $\mathrm{~m}$ & - & 4 \\
\hline 3.01 & 4 & $\mathrm{~m}$ & - & 6,2 \\
\hline 7.10 & 1 & $\mathrm{t}$ & 7.6 & 13 \\
\hline 7.30 & 1 & $\mathrm{~d}$ & 9.8 & 10 \\
\hline 7.35 & 1 & $\mathrm{~d}$ & 7.6 & 12 \\
\hline
\end{tabular}

NMR Spectrometer Varian D.Drive 500 (L4019)

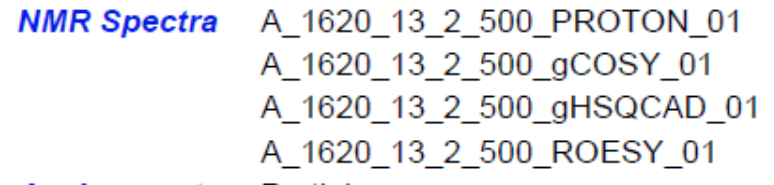

${ }^{1} \mathrm{H}$ NMR (500 MHz, CHLOROFORM- $d$ ) $\delta \mathrm{ppm} 1.18-1.22(\mathrm{~m}, 1 \mathrm{H}) 1.23-1.34(\mathrm{~m}, 2 \mathrm{H}) 1.47-1.57(\mathrm{~m}, 1 \mathrm{H}) 2.18-2.25(\mathrm{~m}, 1 \mathrm{H}) 2.93-3.09(\mathrm{~m}, 4 \mathrm{H}) 7.10$ (t, $J=7.58 \mathrm{~Hz}, 1 \mathrm{H}) 7.30(\mathrm{~d}, J=9.78 \mathrm{~Hz}, 1 \mathrm{H}) 7.35(\mathrm{~d}, J=7.60 \mathrm{~Hz}, 1 \mathrm{H})$

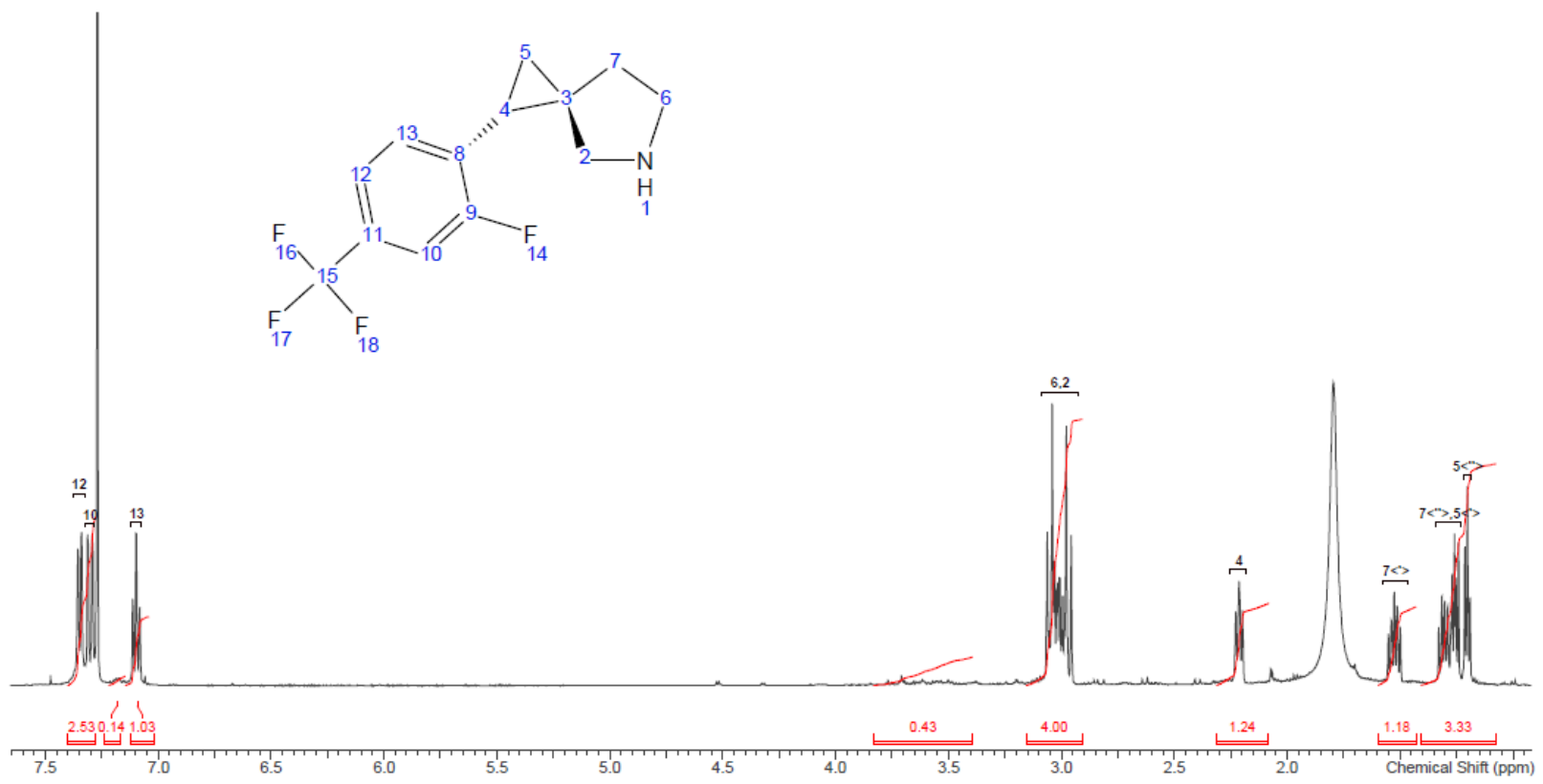




\section{Cis 1-[2-fluoro-4-(trifluoromethyl)phenyl]-5-azaspiro[2.4]heptane}

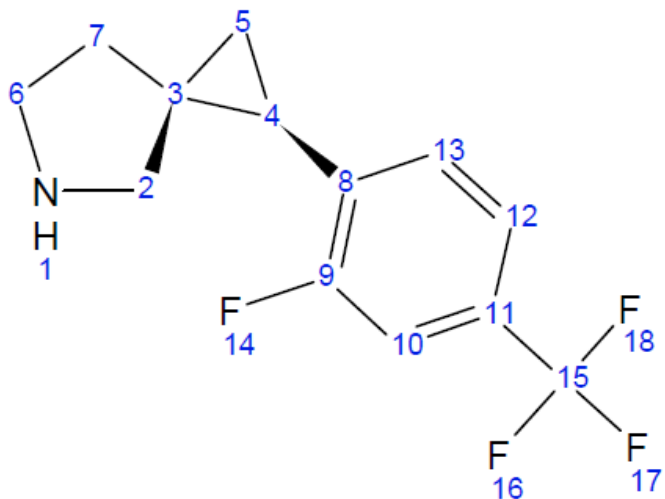

NMR Spectrometer Varian D.Drive 500 (L4019)

16

17
${ }^{1}$ H NMR Assignment

\begin{tabular}{|c|c|c|c|c|}
\hline Shift1 $(\mathrm{ppm})$ & $\mathrm{X}$ 's & Type & $\mathrm{J}(\mathrm{Hz})$ & Atom1 \\
\hline 1.20 & 1 & $\mathrm{t}$ & 5.6 & $5<">$ \\
\hline 1.31 & 1 & $\mathrm{dd}$ & $8.6,5.6$ & $5<\left\langle^{\prime}\right\rangle$ \\
\hline 2.00 & 2 & $\mathrm{~m}$ & - & 7 \\
\hline 2.27 & 1 & $\mathrm{~m}$ & - & 4 \\
\hline 2.47 & 1 & $\mathrm{~d}$ & 11.2 & $\left.2<{ }^{\prime}\right\rangle$ \\
\hline 2.69 & 1 & $\mathrm{~d}$ & 11.2 & $\left.2<{ }^{\prime}\right\rangle$ \\
\hline 3.17 & 2 & $\mathrm{~m}$ & - & 6 \\
\hline 7.09 & 1 & $\mathrm{t}$ & 7.8 & 13 \\
\hline 7.31 & 1 & $\mathrm{~d}$ & 9.8 & 10 \\
\hline 7.35 & 1 & $\mathrm{~d}$ & 8.3 & 12 \\
\hline
\end{tabular}

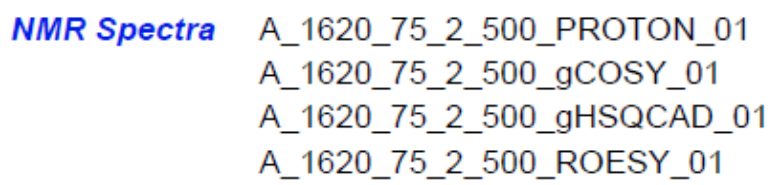

${ }^{1} \mathrm{H} \mathrm{NMR}(500 \mathrm{MHz}$, CHLOROFORM- $d$ ) $\delta$ ppm $1.20(\mathrm{t}, J=5.60 \mathrm{~Hz}, 1 \mathrm{H}) 1.31$ (dd, $J=8.56,5.62 \mathrm{~Hz}, 1 \mathrm{H}) 1.91-2.06$ (m, $\left.2 \mathrm{H}\right) 2.25-2.30$ (m, $\left.1 \mathrm{H}\right) 2.47$ (d, $J=11.25 \mathrm{~Hz}, 1 \mathrm{H}) 2.69(\mathrm{~d}, J=11.25 \mathrm{~Hz}, 1 \mathrm{H}) 3.13-3.19(\mathrm{~m}, 2 \mathrm{H}) 7.09(\mathrm{t}, J=7.83 \mathrm{~Hz}, 1 \mathrm{H}) 7.31(\mathrm{~d}, J=9.78 \mathrm{~Hz}, 1 \mathrm{H}) 7.35(\mathrm{~d}, J=8.31 \mathrm{~Hz}, 1 \mathrm{H})$

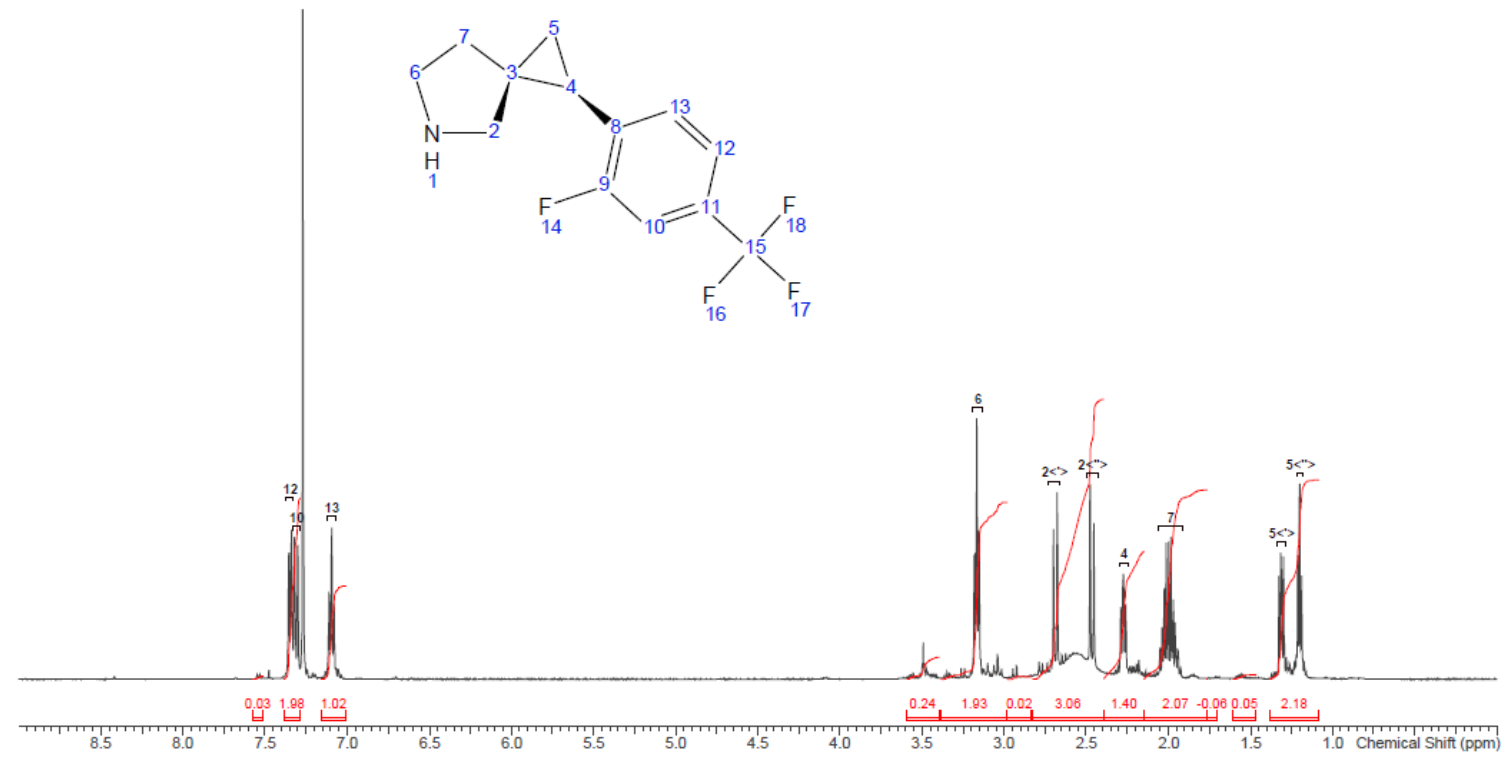




\section{checkCIF/PLATON report}

Structure factors have been supplied for datablock(s) cmpnd-87, cmpnd-89

THIS REPORT IS FOR GUIDANCE ONLY. IF USED AS PART OF A REVIEW PROCEDURE FOR PUBLICATION, IT SHOULD NOT REPLACE THE EXPERTISE OF AN EXPERIENCED CRYSTALLOGRAPHIC REFEREE.

No syntax errors found. CIF dictionary Interpreting this report

\section{Datablock: cmpnd-87}

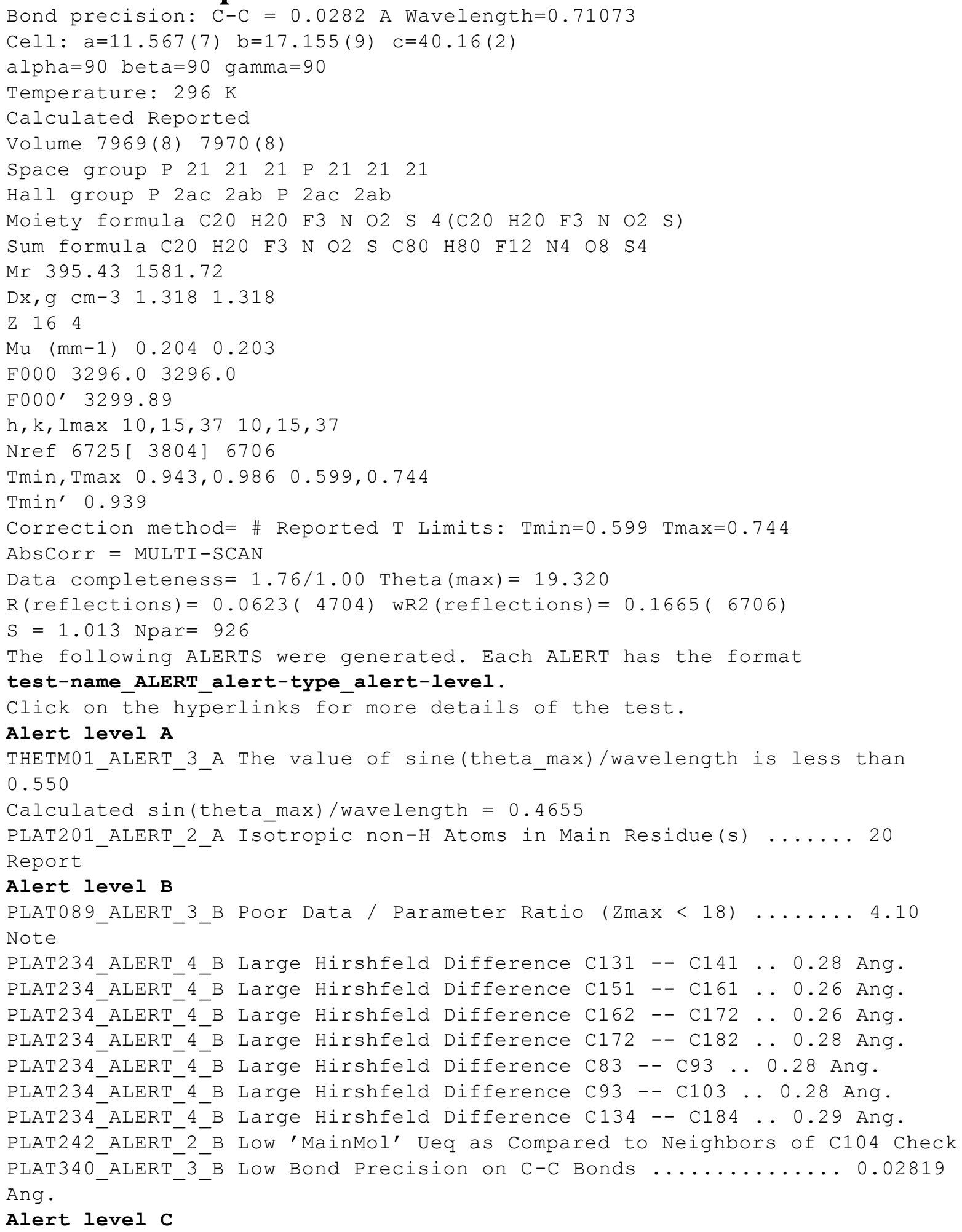

Alert level C 
REFNR01_ALERT_3_C Ratio of reflections to parameters is $<8$ for a non-centrosymmetric structure, where $\mathrm{ZMAX}<18$

sine (theta) / lambda 0.4655

Proportion of unique data used 1.0000

Ratio reflections to parameters 7.2419

PLAT031_ALERT_4_C Refined Extinction Parameter within Range ..... 2.563

Sigma

PLAT220 ALERT 2 C Non-Solvent Resd 2 C Ueq(max)/Ueq(min) Range 3.2 Ratio

PLAT234_ALERT_4_C Large Hirshfeld Difference C171 -- C181 . 0.24 Ang.

PLAT234_ALERT_4_C Large Hirshfeld Difference C12 -- C22 . 0.22 Ang.

PLAT234_ALERT_4_C Large Hirshfeld Difference C22 -- C32 . . 0.18 Ang.

PLAT234_ALERT_4_C Large Hirshfeld Difference C102 -- C112 . 0.22 Ang.

PLAT234_ALERT_4_C Large Hirshfeld Difference C132 -- C182 .. 0.24 Ang.

PLAT234_ALERT_4_C Large Hirshfeld Difference C103 -- C113 .. 0.22 Ang.

PLAT234_ALERT_4_C Large Hirshfeld Difference C133 -- C143 . 0.22 Ang.

PLAT234_ALERT_4_C Large Hirshfeld Difference C14 -- C24 . . 0.21 Ang.

PLAT234_ALERT_4_C Large Hirshfeld Difference C24 -- C34 .. 0.22 Ang.

PLAT234_ALERT_4_C Large Hirshfeld Difference C64 -- C74 .. 0.18 Ang.

PLAT241_ALERT_2_C High 'MainMol' Ueq as Compared to Neighbors of C21 Check PLAT241'ALERT_2_C High 'MainMol' Ueq as Compared to Neighbors of C51 Check PLAT241_ALERT_2_C High 'MainMol' Ueq as Compared to Neighbors of C91 Check PLAT241_ALERT_2_C High 'MainMol' Ueq as Compared to Neighbors of C111

Check

PLAT241_ALERT_2_C High 'MainMol' Ueq as Compared to Neighbors of C52 Check PLAT241_ALERT_2_C High 'MainMol' Ueq as Compared to Neighbors of C92 Check PLAT241_ALERT_2_C High 'MainMol' Ueq as Compared to Neighbors of C202

Check

PLAT241_ALERT_2_C High 'MainMol' Ueq as Compared to Neighbors of C93 Check PLAT241_ALERT_2_C High 'MainMol' Ueq as Compared to Neighbors of C84 Check PLAT241_ALERT_2_C High 'MainMol' Ueq as Compared to Neighbors of C94 Check PLAT241_ALERT_2_C High 'MainMol' Ueq as Compared to Neighbors of C114

Check

PLAT241_ALERT_2_C High 'MainMol' Ueq as Compared to Neighbors of C204 Check

PLAT242_ALERT_2_C Low 'MainMol' Ueq as Compared to Neighbors of C31 Check PLAT242_ALERT_2_C Low 'MainMol' Ueq as Compared to Neighbors of C71 Check PLAT242_ALERT_2_C Low 'MainMol' Ueq as Compared to Neighbors of C101 Check PLAT242_ALERT_2_C Low 'MainMol' Ueq as Compared to Neighbors of C131 Check PLAT242_ALERT_2_C Low 'MainMol' Ueq as Compared to Neighbors of C161 Check PLAT242_ALERT_2_C Low 'MainMol' Ueq as Compared to Neighbors of C72 Check PLAT242_ALERT_2_C Low 'MainMol' Ueq as Compared to Neighbors of C102 Check PLAT242 ALERT_2_C Low 'MainMol' Ueq as Compared to Neighbors of C132 Check PLAT242 ALERT 2 C Low 'MainMol' Ueq as Compared to Neighbors of C162 Check PLAT242_ALERT_2_C Low 'MainMol' Ueq as Compared to Neighbors of C73 Check PLAT242_ALERT_2_C Low 'MainMol' Ueq as Compared to Neighbors of C133 Check PLAT242_ALERT_2_C Low 'MainMol' Ueq as Compared to Neighbors of C74 Check PLAT242_ALERT_2_C Low 'MainMol' Ueq as Compared to Neighbors of C134 Check PLAT334_ALERT_2_C Small Average Benzene C-C Dist. C71 -C121 1.36 Ang. PLAT334_ALERT_2_C Small Average Benzene C-C Dist. C131-C181 1.37 Ang. PLAT334_ALERT_2_C Small Average Benzene C-C Dist. C132 -C182 1.36 Ang. PLAT334_ALERT_2_C Small Average Benzene C-C Dist. C73-C123 1.37 Ang. PLAT334_ALERT_2_C Small Average Benzene C-C Dist. C133-C183 1.37 Ang. PLAT334_ALERT_2_C Small Average Benzene C-C Dist. C74-C1241.36 Ang. PLAT334 ALERT 2 C Small Average Benzene C-C Dist. C134-C184 1.37 Ang. PLAT911_ALERT_3_C Missing \# FCF Refl Between THmin \& STh/L=0.466 9 Report PLAT978_ALERT_2_C Number C-C Bonds with Positive Residual Density 0 Note

Alert level $\mathbf{G}$

PLAT002_ALERT_2_G Number of Distance or Angle Restraints on Atsite 32 Note 
PLAT042_ALERT_1_G Calc. and Reported MoietyFormula Strings Differ Please Check

PLAT045_ALERT_1_G Calculated and Reported Z Differ by a Factor ... 4.00

Check

PLAT083_ALERT_2_G SHELXL Second Parameter in WGHT Unusually Large 9.40 Why ?

PLAT172_ALERT_4_G The CIF-Embedded.res File Contains DFIX Records 10

Report

PLAT173 ALERT 4 G The CIF-Embedded. res File Contains DANG Records 8

Report

PLAT242_ALERT_2_G Low 'MainMol' Ueq as Compared to Neighbors of C203 Check

PLAT301_ALERT_3_G Main Residue Disorder......... Percentage = 11 Note

PLAT367_ALERT_2_G Long? C (sp?) -C (sp?) Bond C103 - C203 . . 1.59 Ang.

PLAT367_ALERT_2_G Long? C (sp?) -C (sp?) Bond C104 - C204 .. 1.56 Ang.

PLAT380_ALERT_4_G Incorrectly? Oriented X(sp2)-Methyl Moiety .... C191

Check

PLAT380_ALERT_4_G Incorrectly? Oriented X(sp2)-Methyl Moiety .... C192

Check

PLAT380_ALERT_4_G Incorrectly? Oriented X(sp2)-Methyl Moiety .... C193

Check

PLAT380 ALERT 4 G Incorrectly? Oriented X(sp2)-Methyl Moiety .... C194

Check

PLAT791_ALERT_4_G The Model has Chirality at C31 (Chiral SPGR) R Verify

PLAT791_ALERT_4_G The Model has Chirality at C32 (Chiral SPGR) R Verify

PLAT791_ALERT_4_G The Model has Chirality at C33 (Chiral SPGR) R Verify

PLAT791_ALERT_4_G The Model has Chirality at C34 (Chiral SPGR) R Verify

PLAT791_ALERT_4_G The Model has Chirality at C61 (Chiral SPGR) S Verify

PLAT791_ALERT_4_G The Model has Chirality at C62 (Chiral SPGR) S Verify

PLAT791_ALERT_4_G The Model has Chirality at C63 (Chiral SPGR) S Verify

PLAT791_ALERT_4_G The Model has Chirality at C64 (Chiral SPGR) S Verify

PLAT860_ALERT_3_G Number of Least-Squares Restraints .......... 56 Note

PLAT909ALERT_3_G Percentage of Observed Data at Theta (Max) Still $42 \%$

PLAT910 ALERT_3_G Missing \# of FCF Reflection(s) Below Theta(Min) 1 Note

2 ALERT level $\mathbf{A}=$ Most likely a serious problem - resolve or explain

10 ALERT level $\mathbf{B}=\mathrm{A}$ potentially serious problem, consider carefully

47 ALERT level $\mathbf{C}=$ check. Ensure it is not caused by an omission or

oversight

25 ALERT level $\mathbf{G}=$ General information/check it is not something unexpected

2 ALERT type 1 CIF construction/syntax error, inconsistent or missing data 41 ALERT type 2 Indicator that the structure model may be wrong or deficient

9 ALERT type 3 Indicator that the structure quality may be low

32 ALERT type 4 Improvement, methodology, query or suggestion

0 ALERT type 5 Informative message, check

\section{Datablock: cmpnd-89}

Bond precision: $\mathrm{C}-\mathrm{C}=0.0462 \mathrm{~A}$ Wavelength $=0.71073$

Cell: $a=11.75(5) \quad b=17.30(7) \quad c=41.04$ (9)

alpha $=90$ beta $=90$ gamma $=90$

Temperature: $296 \mathrm{~K}$

Calculated Reported

Volume $8342(52) 8342(52)$

Space group P $2121 \quad 21 \quad$ P 212121

Hall group P 2ac $2 \mathrm{ab}$ P $2 \mathrm{ac} 2 \mathrm{ab}$

Moiety formula C20 H20 F3 N O2 S $4($ C20 H20 F3 N O2 S)

Sum formula C20 H20 F3 N O2 S C80 H80 F12 N4 O8 S4

$\operatorname{Mr} 395.43 \quad 1581.72$ 
Dx, $g \quad c m-3 \quad 1.2591 .259$

Z 164

$\mathrm{Mu}(\mathrm{mm}-1) \quad 0.194 \quad 0.194$

F000 $3296.0 \quad 3296.0$

F000' 3299.89

h, k, lmax 9,14,34 9,14,33

Nref 5073[ 2903] 4938

Tmin, Tmax $0.951,0.9960 .346,0.744$

Tmin' 0.951

Correction method= \# Reported T Limits: Tmin=0.346 Tmax=0.744

AbsCorr = MULTI-SCAN

Data completeness $=1.70 / 0.97$ Theta $(\max )=17.238$

$R($ reflections $)=0.0786(2435) \quad w R 2($ reflections $)=0.2100(4938)$

$\mathrm{S}=1.003$ Npar $=686$

The following ALERTS were generated. Each ALERT has the format

\section{test-name_ALERT_alert-type_alert-level.}

Click on the hyperlinks for more details of the test.

\section{Alert level A}

THETM01_ALERT_3_A The value of sine(theta_max)/wavelength is less than 0.550

Calculated sin(theta_max)/wavelength $=0.4170$

PLAT201_ALERT_2_A Isotropic non-H Atoms in Main Residue(s) ..... 68 Report

PLAT234_ALERT_4_A Large Hirshfeld Difference C31 -- C61 . 0.35 Ang.

PLAT234_ALERT_4_A Large Hirshfeld Difference C33 -- C53 . . 0.32 Ang.

PLAT241_ALERT_2_A High 'MainMol' Ueq as Compared to Neighbors of C202

Check

PLAT241_ALERT_2_A High 'MainMol' Ueq as Compared to Neighbors of C203

Check

PLAT241_ALERT_2_A High 'MainMol' Ueq as Compared to Neighbors of C204

Check

\section{Alert level B}

RINTA01_ALERT_3_B The value of Rint is greater than 0.18

Rint given $0 . \overline{1} 8 \overline{2}$

PLAT020_ALERT_3_B The value of Rint is greater than $0.12 \ldots \ldots .182$

Report

PLAT031_ALERT_4_B Refined Extinction Parameter within Range ..... 1.500

Sigma

PLAT089_ALERT_3_B Poor Data / Parameter Ratio (Zmax < 18) .....4.14

Note

PLAT213_ALERT_2_B Atom $\mathrm{C} 61$ has ADP max/min Ratio.... 4.6 prolat

PLAT213_ALERT_2_B Atom $\mathrm{Cl} 4$ has ADP max/min Ratio.... 4.1 prolat

PLAT234_ALERT_4_B Large Hirshfeld Difference C52 -- C62 . . 0.30 Ang.

PLAT234_ALERT_4_B Large Hirshfeld Difference C14 -- C24 . . 0.28 Ang.

PLAT242_ALERT_2_B Low 'MainMol' Ueq as Compared to Neighbors of C101 Check

PLAT242_ALERT_2_B Low 'MainMol' Ueq as Compared to Neighbors of C102 Check

PLAT242_ALERT_2_B Low 'MainMol' Ueq as Compared to Neighbors of C103 Check PLAT242_ALERT_2_B Low 'MainMol' Ueq as Compared to Neighbors of C104 Check PLAT340_ALERT_3 ${ }^{-}$B LOW Bond Precision on C-C Bonds ............0.04619 Ang.

\section{Alert level $C$}

REFNR01_ALERT_3_C Ratio of reflections to parameters is $<8$ for a non-centrosymmetric structure, where ZMAX $<18$

sine (theta) / lambda 0.4170

Proportion of unique data used 1.0000

Ratio reflections to parameters 7.1983

PLAT026_ALERT_3_C Ratio Observed / Unique Reflections (too) Low .. 49 \% 
PLAT029_ALERT_3_C_diffrn_measured_fraction_theta_full value Low . 0.979 Note

PLAT148_ALERT_3_C s.u. on the a - Axis is (Too) Large ..... 0.050 Ang. PLAT148_ALERT_3_C s.u. on the b - Axis is (TOO) Large .....0.0700 Ang. PLAT148_ALERT_3_C s.u. on the c - Axis is (TOO) Large ..... 0.090 Ang. PLAT213_ALERT_2_C Atom C11 has ADP max/min Ratio.... 3.3 oblate PLAT213_ALERT_2_C Atom $\mathrm{C} 52$ has ADP max/min Ratio..... 3.6 oblate PLAT213 ALERT 2 C Atom $\mathrm{C} 23$ has ADP max/min Ratio..... 3.7 oblate PLAT220_ALERT_2 C Non-Solvent Resd 2 C Ueq(max)/Ueq(min) Range 4.6 Ratio PLAT220_ALERT_2_C Non-Solvent Resd 2 F Ueq(max)/Ueq(min) Range 3.9 Ratio PLAT220_ALERT_2_C Non-Solvent Resd 3 C Ueq(max)/Ueq(min) Range 4.8 Ratio PLAT220_ALERT_2_C Non-Solvent Resd 4 C Ueq(max)/Ueq(min) Range 5.8 Ratio PLAT222_ALERT_3_C Non-Solvent Resd 2 H Uiso(max)/Uiso(min) Range 4.9 Ratio PLAT241_ALERT_2_C High 'MainMol' Ueq as Compared to Neighbors of C11 Check PLAT241_ALERT_2_C High 'MainMol' Ueq as Compared to Neighbors of C51 Check PLAT241_ALERT_2 C High 'MainMol' Ueq as Compared to Neighbors of C111 Check

PLAT241_ALERT_2_C High 'MainMol' Ueq as Compared to Neighbors of C201 Check

PLAT241_ALERT_2_C High 'MainMol' Ueq as Compared to Neighbors of C52 Check PLAT241_ALERT_2_C High 'MainMol' Ueq as Compared to Neighbors of C82 Check PLAT241_ALERT_2_C High 'MainMol' Ueq as Compared to Neighbors of C122

Check

PLAT241_ALERT_2_C High 'MainMol' Ueq as Compared to Neighbors of C23 Check PLAT241_ALERT_2_C High 'MainMol' Ueq as Compared to Neighbors of C83 Check PLAT241_ALERT_2_C High 'MainMol' Ueq as Compared to Neighbors of C14 Check PLAT241_ALERT_2_C High 'MainMol' Ueq as Compared to Neighbors of C44 Check PLAT241_ALERT_2_C High 'MainMol' Ueq as Compared to Neighbors of C84 Check PLAT241_ALERT_2_C High 'MainMol' Ueq as Compared to Neighbors of C124 Check

PLAT241_ALERT_2_C High 'MainMol' Ueq as Compared to Neighbors of C144 Check

PLAT242_ALERT_2_C Low 'MainMol' Ueq as Compared to Neighbors of N11 Check PLAT242_ALERT_2_C Low 'MainMol' Ueq as Compared to Neighbors of C71 Check PLAT242_ALERT_2_C Low 'MainMol' Ueq as Compared to Neighbors of C131 Check PLAT242_ALERT_2_C Low 'MainMol' Ueq as Compared to Neighbors of C161 Check PLAT242_ALERT_2_C Low 'MainMol' Ueq as Compared to Neighbors of C62 Check PLAT242_ALERT_2_C Low 'MainMol' Ueq as Compared to Neighbors of C72 Check PLAT242_ALERT_2_C Low 'MainMol' Ueq as Compared to Neighbors of C132 Check PLAT242_ALERT_2_C Low 'MainMol' Ueq as Compared to Neighbors of C162 Check PLAT242_ALERT_2_C Low 'MainMol' Ueq as Compared to Neighbors of C43 Check PLAT242_ALERT_2_C Low 'MainMol' Ueq as Compared to Neighbors of C73 Check PLAT242 ALERT 2 C Low 'MainMol' Ueq as Compared to Neighbors of C93 Check PLAT242_ALERT_2_C Low 'MainMol' Ueq as Compared to Neighbors of C133 Check PLAT242_ALERT_2_C Low 'MainMol' Ueq as Compared to Neighbors of N14 Check PLAT242_ALERT_2_C Low 'MainMol' Ueq as Compared to Neighbors of C74 Check PLAT242_ALERT_2_C Low 'MainMol' Ueq as Compared to Neighbors of C114 Check PLAT242_ALERT_2_C Low 'MainMol' Ueq as Compared to Neighbors of C134 Check PLAT242_ALERT_2_C Low 'MainMol' Ueq as Compared to Neighbors of C154 Check PLAT250_ALERT_2_C Large U3/U1 Ratio for Average U(i,j) Tensor ... 2.2 Note

PLAT250_ALERT_2_C Large U3/U1 Ratio for Average U(i,j) Tensor ... 2.8 Note

PLAT250_ALERT_2_C Large U3/U1 Ratio for Average U(i,j) Tensor .... 3.3 Note

PLAT250_ALERT_2_C Large U3/U1 Ratio for Average U(i,j) Tensor.... 2.9 Note

PLAT334_ALERT_2_C Small Average Benzene C-C Dist. C134-C184 1.37 Ang. 
PLAT360_ALERT_2_C Short C (sp3)-C(sp3) Bond C32 - C52 . 1.42 Ang. PLAT362_ALERT_2_C Short C (sp3)-C(sp2) Bond C61 - C71 . 1.39 Ang. PLAT363_ALERT_2_C Long C (sp3)-C(sp2) Bond C163 - C193 .. 1.65 Ang. PLAT911_ALERT_3_C Missing \# FCF Refl Between THmin \& STh/L=0.41759 Report

PLAT978_ALERT_2_C Number C-C Bonds with Positive Residual Density 0 Note Alert level G

PLAT002_ALERT_2_G Number of Distance or Angle Restraints on AtSite 32 Note PLAT003_ALERT_2_G Number of Uiso or Uij Restrained non-H Atoms ... 4 Report

PLAT042_ALERT_1_G Calc. and Reported MoietyFormula Strings Differ Please Check

PLAT045_ALERT_1_G Calculated and Reported Z Differ by a Factor ... 4.00

Check

PLAT172_ALERT_4_G The CIF-Embedded.res File Contains DFIX Records 10 Report

PLAT173_ALERT_4_G The CIF-Embedded .res File Contains DANG Records 8 Report

PLAT178_ALERT_4_G The CIF-Embedded.res File Contains SIMU Records 1 Report

PLAT301_ALERT_3_G Main Residue Disorder........... Percentage = 11 Note PLAT335_ALERT_2_G Check Large C6 Ring C-C Range C132 -C182 0.20 Ang. PLAT335_ALERT_2_G Check Large C6 Ring C-C Range C133-C183 0.19 Ang. PLAT343_ALERT_2_G Unusual sp3 Angle Range in Main Residue for C32 Check PLAT343_ALERT_2_G Unusual sp3 Angle Range in Main Residue for C33 Check PLAT380_ALERT_4_G Incorrectly? Oriented X(sp2)-Methyl Moiety .... C192 Check

PLAT380_ALERT_4_G Incorrectly? Oriented X(sp2)-Methyl Moiety .... C193 Check

PLAT380_ALERT_4_G Incorrectly? Oriented X(sp2)-Methyl Moiety .... C194 Check

PLAT721 ALERT 1 G Bond CalC 0.96000, Rep 0.97000 Dev... 0.01 Ang. C53 - H5 $\overline{3} \mathrm{~A} 1.5 \overline{5} 51.555 \ldots$....... Bond \# 86 Check

PLAT721_ALERT_1_G Bond CalC 0.96000, Rep 0.97000 Dev... 0.01 Ang.

C52 - $55 \overline{2} \mathrm{~B} 1.5 \overline{5} 51.555 \ldots . .$. Bond \# 94 Check

PLAT721_ALERT_1_G Bond Calc 0.97000, Rep 0.96000 Dev... 0.01 Ang.

C194-H19F $1 . \overline{5} 5 \overline{5} 1.555 \ldots \ldots$ Bond \# 182 Check

PLAT721_ALERT_1_G Bond CalC 0.97000, Rep 0.96000 Dev... 0.01 Ang. C191-H19L $1 . \overline{5} 5 \overline{5} 1.555 \ldots . . .$. Bond \# 188 Check

PLAT791_ALERT_4_G The Model has Chirality at C31 (Chiral SPGR) S Verify PLAT791_ALERT_4_G The Model has Chirality at C32 (Chiral SPGR) S Verify PLAT791_ALERT_4_G The Model has Chirality at C33 (Chiral SPGR) S Verify PLAT791 ALERT 4 G The Model has Chirality at C34 (Chiral SPGR) S Verify PLAT791_ALERT_4_G The Model has Chirality at C61 (Chiral SPGR) R Verify PLAT791 ALERT 4 G The Model has Chirality at C62 (Chiral SPGR) R Verify PLAT791_ALERT_4_G The Model has Chirality at C63 (Chiral SPGR) R Verify PLAT791_ALERT_4_G The Model has Chirality at C64 (Chiral SPGR) R Verify PLAT860 ALERT 3 G Number of Least-Squares Restraints ......... 74 Note PLAT910 ALERT 3_G Missing \# of FCF Reflection(s) Below Theta(Min) 1 Note

7 ALERT level $\mathbf{A}^{-}=$Most likely a serious problem - resolve or explain

13 ALERT level $\mathbf{B}=$ A potentially serious problem, consider carefully

55 ALERT level $\mathbf{C}=$ Check. Ensure it is not caused by an omission or oversight

29 ALERT level $\mathbf{G}=$ General information/check it is not something unexpected

6 ALERT type 1 CIF construction/syntax error, inconsistent or missing data 63 ALERT type 2 Indicator that the structure model may be wrong or deficient 
16 ALERT type 3 Indicator that the structure quality may be low

19 ALERT type 4 Improvement, methodology, query or suggestion

0 ALERT type 5 Informative message, check

It is advisable to attempt to resolve as many as possible of the alerts in all categories. Often the minor alerts point to easily fixed oversights, errors and omissions in your CIF or refinement strategy, so attention to these fine details can be worthwhile. In order to resolve some of the more serious problems it may be necessary to carry out additional measurements or structure refinements. However, the purpose of your study may justify the reported deviations and the more serious of these should normally be commented upon in the discussion or experimental section of a paper or in the "special_details" fields of the CIF. checkCIF was carefully designed to identify outliers and unusual parameters, but every test has its limitations and alerts that are not important in a particular case may appear. Conversely, the absence of alerts does not guarantee there are no aspects of the results needing attention. It is up to the individual to critically assess their own results and, if necessary, seek expert advice.

\section{Publication of your CIF in IUCr journals}

A basic structural check has been run on your CIF. These basic checks will be run on all CIFs submitted for publication in IUCr journals (Acta Crystallographica, Journal of Applied Crystallography, Journal of Synchrotron Radiation); however, if you intend to submit to Acta Crystallographica Section $C$ or $E$ or IUCrData, you should make sure that full publication checks

are run on the final version of your CIF prior to submission.

\section{Publication of your CIF in other journals}

Please refer to the Notes for Authors of the relevant journal for any special instructions relating to

CIF submission.

PLATON version of 06/05/2016; check.def file version of $05 / 05 / 2016$ 
Datablock cmpnd-87 - ellipsoid plot

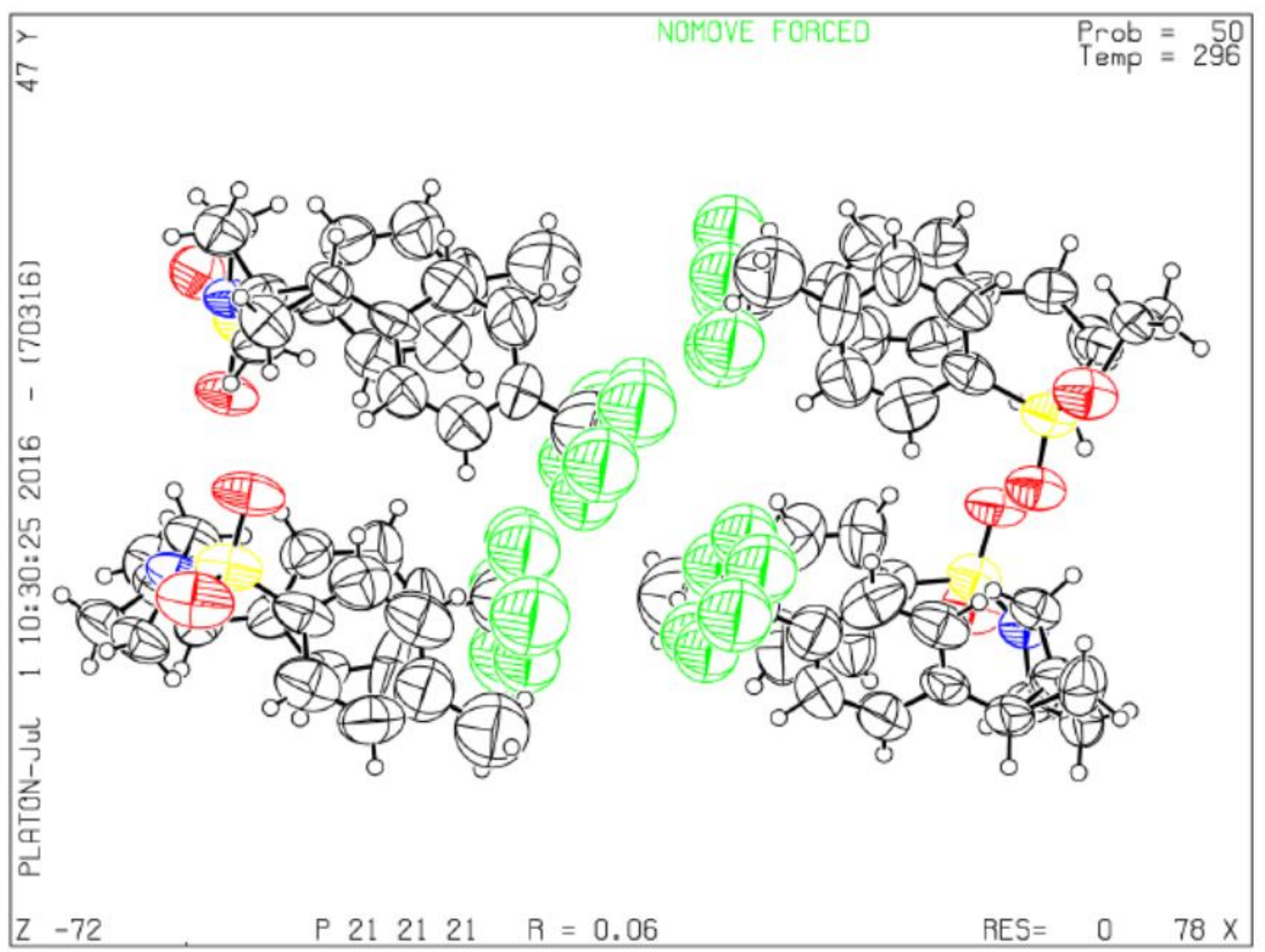


Datablock cmpnd-89 - ellipsoid plot

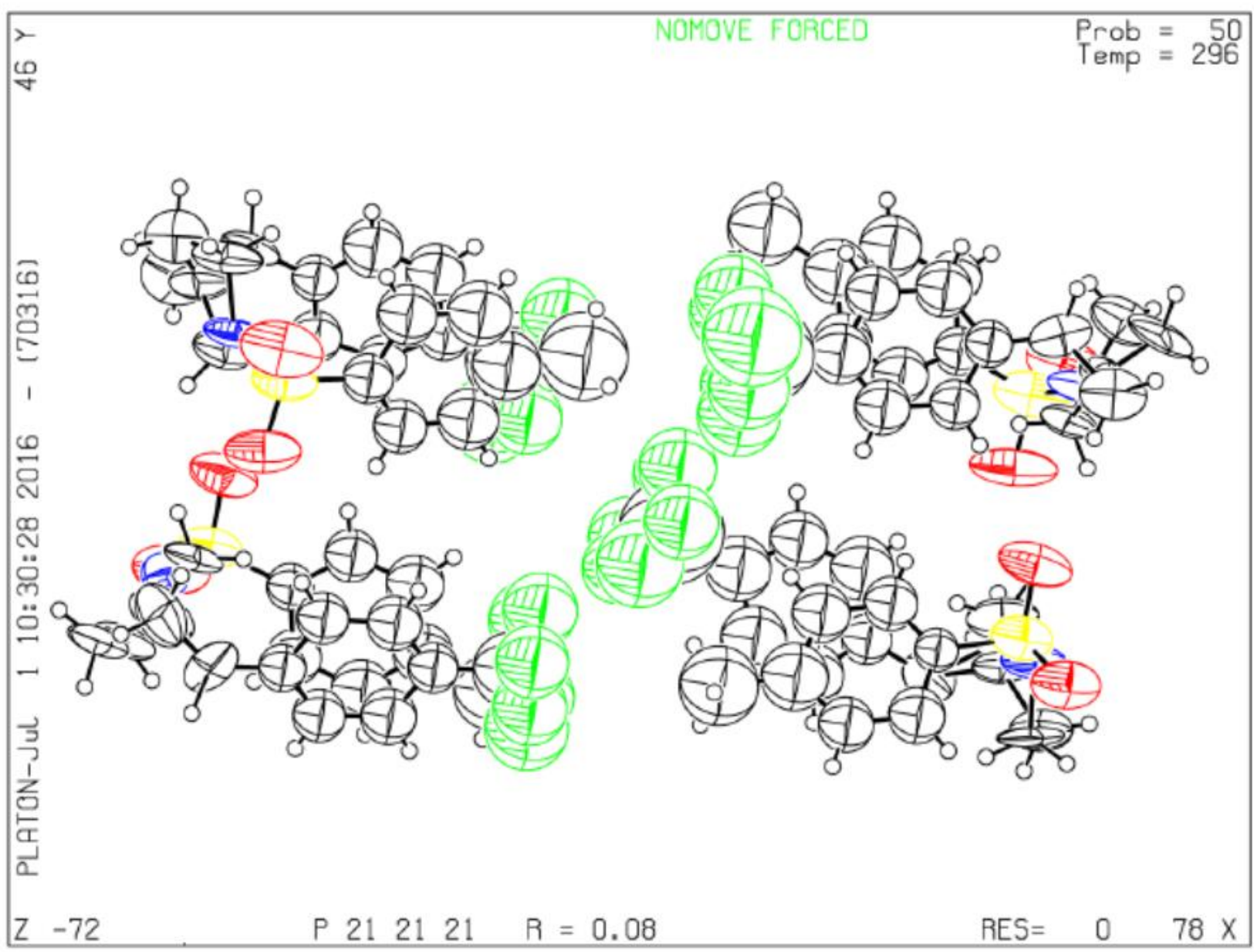


